\title{
The Gene Polymorphism of VMAT2 Is Associated with Risk of Schizophrenia in Male Han Chinese
}

\author{
Hongying $\mathrm{Han}^{1 *}$, Xiaowei Xia ${ }^{1 *}$, Huirong Zheng ${ }^{2}$, Chongbang Zhao', \\ Yanming $\mathrm{Xu}^{3}$, Jiong $\mathrm{Tao}^{1 凶}$, and Xianglan Wang ${ }^{1 凶}$ \\ ${ }^{1}$ Department of Psychiatry, The Third Affiliated Hospital of Sun Yat-sen University, Guangzhou, China \\ ${ }^{2}$ Guangdong Mental Health Center, Guangdong Provincial People's Hospital, Guangdong Academy of Medical Sciences, \\ Affiliated School of Medicine of South China University of Technology, Guangzhou, China \\ ${ }^{3}$ Department of Neurology, West China Hospital, Sichuan University, Chengdu, China
}

Objective To investigate the association between gene polymorphism of vesicular monoamine transporter type 2(VMAT2) and schizophrenia in Han Chinese population.

Methods 430 patients with schizophrenia and 470 age-sex matched controls were recruited from four mental health centers. All patients were diagnosed by two psychiatrists based on the Structured Clinical Interview for DSM Disorders (SCID). The ligase detection reactions (LDR) method was used to assess the polymorphism of the two SNPs (rs363371 and rs363324) of VMAT2.

Results No associations of two SNPs with schizophrenia was found. When we stratified males and females for the analysis, we found that that in the recessive model of rs363371, there was an obvious significant association between rs363371 and schizophrenia in males $(\mathrm{OR}=0.564,95 \% \mathrm{CI}=0.357-0.892, \mathrm{p}=0.014)$ but not females. For the association between rs 363324 and schizophrenia, no association was found in either males or females. No association was found when stratifying early-onset schizophrenia and late-onset schizophrenia.

Conclusion Our findings indicate that both rs363371 and rs363324 were not associated with schizophrenia, while it seemed that the AA genotype of rs363371 plays a protective effect in male Chinese in developing schizophrenia.

Psychiatry Investig 2020;17(11):1073-1078

Key Words Schizophrenia, VMAT2, Gene polymorphism, Male, Han Chinese.

\section{INTRODUCTION}

Schizophrenia (SCZ) is a severe psychiatric disorder with a prevenlance of $1 \%$ worldwide. Schizophrenia is a complex disease which many genes and environmental factors are involved in its pathogenesis. ${ }^{1}$ Its etiology is still unclear. Among numerous physiologic mechanism of Schizophrenia, dopa-

Received: January 27, 2020 Revised: May 2, 2020

Accepted: June 11, 2020

$\triangle$ Correspondence: Jiong Tao, MD

Department of Psychiatry, The Third Affiliated Hospital of Sun Yat-sen University, 600 Tianhe Road, Guangzhou, Guangdong Province 510630, China Tel: +86-20-85252190, Fax: +86-20-85252190, E-mail: tj2023@163.com

$\bowtie$ Correspondence: Xianglan Wang, MD

Department of Psychiatry, The Third Affiliated Hospital of Sun Yat-sen University, 600 Tianhe Road, Guangzhou, Guangdong Province 510630, China

Tel: +86-20-85252190, Fax: +86-20-85252190, E-mail: wxl0372@126.com

*These authors contributed equally to this work.

(a) This is an Open Access article distributed under the terms of the Creative Commons Attribution Non-Commercial License (https://creativecommons.org/licenses/bync/4.0) which permits unrestricted non-commercial use, distribution, and reproduction in any medium, provided the original work is properly cited. mine hypothesis is the most accepted one which suggests excessive activity of dopamine function or drugs increasing dopamine function may induce psychotic symptoms. And in clinical practice, the dopaminergic receptor antagonist (e.g., chlorpromazine, clozapine, risperidone, etc) were extensively used to treat Schizophrenia.

Many GWAS studies and related studies have detected genetic factors may be associated with SCZ, which indicates a genetic background of schizophrenia. ${ }^{2-4}$ One of these genes is the VMAT2 gene, which is located at 10q25. A suspected locus of schizophrenia detected by a systematic genome wide linkage study had a LOD score of $3.87 .{ }^{5}$ VMAT2 is an integral membrane protein that transports monoamine from the cytoplasm to the vesicles. ${ }^{6}$ VMAT2 protein mainly expresses in the brain and plays an important role in the transmission of monoamineC: \Users \Administrator $\backslash A p p D a t a \backslash$ Local $\backslash$ Yodao\DeskDict\frame\20140816171600 \javascript:void(0); including dopamine. 
Table 1. Demographic characteristics of Han Chinese with schizophrenia (SCZ) and healthy controls

\begin{tabular}{lccc}
\multicolumn{1}{c}{ Characteristic } & $\operatorname{SCZ}(\mathrm{n}=430)$ & Controls $(\mathrm{n}=470)$ & Comparison* \\
\hline Age years, mean \pm SD & $41.27 \pm 14.40$ & $42.33 \pm 12.45$ & $\mathrm{t}=0.18, \mathrm{p}=0.24$ \\
$\mathrm{Sex}(\mathrm{N})$ & 185 & 190 & $0.80,0.63-1.02,3.14,0.08$ \\
Male & 245 & 280 & \\
Female & & & \\
\hline
\end{tabular}

*unless otherwise indicated, the values indicate: odds ratio, $95 \%$ confidence interval, $\chi^{2}$, $p$ value. SD: standard deviation

Recently, an Italian study reported that two SNPs (rs363371, rs363324) in the promoter region of VMAT2 are associated with Parkinson's disease. ${ }^{7}$ Since dopamine hypothesis was the common base of both Schizophrenia and Parkinson's disease. For patients with Parkinson's disease, the deficiency of dopamine in substantia nigra accounts for the main clinical characteristics of the motor symptoms in Parkinsonism. Nevertheless, the new revised dopamine hypothesis of schizophrenia is the imbalance of dopamine function in different brain regions, which is the hyperactive dopamine transmission in the mesolimbic areas but hypoactive dopamine transmission in the prefrontal cortex. ${ }^{8}$ Interestingly, both Schizophrenia and Parkinson's disease have shared some common genetic risk factors such as MMP9, ${ }^{9,10}$ MAOB, COMT, etc. ${ }^{11-13}$

However, it has not been clear the association of VMAT2 SNP with schizophrenia. Thus, as the shared pathogenic mechanism and genetic factors between schizophrenia and Parkinson's disease, we conducted a case-control study to explore the association between VMAT2 gene polymorphism and Schizophrenia in Han Chinese population.

\section{METHODS}

\section{Subjects}

A total of 430 (male185 43.02\%); mean age $41.27 \pm 14.40$ years, mean onset age $25.05 \pm 8.69$ years) patients with SCZ were recruited from four psychiatric disorder centers: The Second Xiangya Hospital of Central South University, located in central China; West China Hospital of Sichuan University, located in southwest China; and the Third Affiliated Hospital of Sun Yat-sen University and Jining Mental Health Hospital, both located in east China. All patients were diagnosed by two psychiatrists based on the Structured Clinical Interview for DSM Disorders (SCID) and DSM-IV criteria. ${ }^{14}$ Patients who were older than 18 at SCZ onset were subdivided into late-onset SCZ (LOSCZ; n=290; mean age at onset, 27.71 \pm 7.96 years); the others were as early-onset SCZ group (EOSCZ; n=140; mean age at onset, $15.57 \pm 2.05$ years). ${ }^{15}$ The control group for SCZ patients comprised 457 Han Chinese (190 males, 280 females; mean age, $42.33 \pm 12.45$ years). The protocol of the study was approved by the Ethics Committees of all four hospitals involved in this research (No [2015]2-23), all the written informed consents were obtained from all the subjects involved in this study.

\section{Genotyping}

Genomic DNA was obtained from peripheral leukocytes by the classic Phenol-chloroform DNA extract method. All the genotyping experiments were done by the Shanghai BioWing Applied Biotechnology Company using ligase detection reactions (LDR). ${ }^{16}$ The target DNA sequences were amplified using a multiplex PCR method. The primary information for rs363371 and rs363324 listed in Supplementary Table 1 (in the online-only Data Supplement). After the completion of the amplification, $1 \mu \mathrm{L}$ of Proteinase $\mathrm{K}(20 \mathrm{mg} / \mathrm{mL})$ was added, then heated at $70^{\circ} \mathrm{C}$ for $10 \mathrm{~min}$ and quenched at $94^{\circ} \mathrm{C}$ for 15 $\mathrm{min}$. The ligation reaction for each subject was carried out in a final volume of $20 \mu \mathrm{L}$ containing $20 \mathrm{mM}$ Tris- $\mathrm{HCl}$ ( $\mathrm{pH} 7.6$ ), 25 $\mathrm{mM}$ potassium acetate, $10 \mathrm{mM}$ magnesium acetate, $10 \mathrm{mM}$ DTT, $1 \mathrm{mM}$ NAD, $0.1 \%$ Triton X-100, $10 \mu \mathrm{L}$ of MultiPCR product, 1 pmol of each discriminating oligo, 1 pmol of each common probe and $0.5 \mu \mathrm{L}$ of $40 \mathrm{U} / \mu \mathrm{L}$ Taq DNA ligase (New England Biolabs, USA). The LDR was performed using $40 \mathrm{cy}-$ cles of $94^{\circ} \mathrm{C}$ for $30 \mathrm{~s}$ and $63^{\circ} \mathrm{C}$ for $4 \mathrm{~min}$. The fluorescent products of LDR were differentiated by $\mathrm{ABI}$ sequencer 377 .

\section{Statistical analysis}

Values for age were represented by the mean \pm standard deviation (SD), and the gender, allele and genotype were described as percentages. Allele and genotype frequencies were determined by direct counting of VMAT2 alleles. Concordance of genotype distribution was verified by Hardy-Weinberg equilibrium (HWE) and then evaluated with the chisquare test. Associations between gender, allele and genotype were also assessed by the chi-square test. Age differences between the three groups were assessed by the t-test. A twotailed $\mathrm{p}$ value $<0.025$ was considered significant. Power calculations were carried out using "Power and Sample Size Program." We recruited a study with 430 case patients and 470 control patients. Prior data indicated that the probability of exposure among controls was 0.302 . If the true odds ratio for disease in exposed subjects relative to unexposed subjects was 0.284 , we rejected the null hypothesis that this odds ratio 
Table 2. Genotype frequencies between the entire group or subgroups of Han Chinese with SCZ and healthy controls*

\begin{tabular}{|c|c|c|c|c|c|}
\hline \multirow{2}{*}{$\begin{array}{l}\text { Group or } \\
\text { subgroup }\end{array}$} & \multicolumn{3}{|c|}{ Genotype, N (\%) } & \multicolumn{2}{|c|}{ Comparison $^{\dagger}$} \\
\hline & GG & AG & AA & Dominant model & Recessive model \\
\hline \multicolumn{6}{|l|}{ Locus rs363371 } \\
\hline All SCZ & $81(18.8)$ & $227(52.8)$ & $122(28.4)$ & $1.607,0.761-1.495,0.14,0.709^{\ddagger}$ & $0.915,0.686-1.22,0.367,0.545^{\ddagger}$ \\
\hline Male SCZ & $32(17.3)$ & $111(60)$ & $42(22.7)$ & \multirow{2}{*}{$1.013,0.591-1.736,0.002,0.962$} & \multirow{2}{*}{$0.564,0.357-0.892,6.059,0.014^{\S}$} \\
\hline Male controls & $32(17.1)$ & $91(48.7)$ & $64(34.2)$ & & \\
\hline Female SCZ & $49(20)$ & $116(47.3)$ & $80(32.7)$ & \multirow{2}{*}{$1.239,0.796-1.930,0.903,0.342$} & \multirow{2}{*}{$1.112,0.769-1.609,0.32,0.572^{\S}$} \\
\hline Female controls & $47(16.8)$ & $148(52.8)$ & $85(30.4)$ & & \\
\hline EOSCZ & $26(18.7)$ & $80(57.6)$ & $33(23.7)$ & $1.057,0.65-1.721,0.05,0.823$ & $0.719,0.464-1.114,2.194,0.139$ \\
\hline LOSCZ & $55(18.9)$ & $147(50.5)$ & $89(30.6)$ & $1.071,0.735-1.561,0.127,0.721$ & $1.018,0.741-1.398,0.012,0.914$ \\
\hline All controls & $84(17.9)$ & $244(51.9)$ & $142(30.2)$ & & \\
\hline \multicolumn{6}{|l|}{ Locus rs363324 } \\
\hline SCZ & $265(61.6)$ & $147(34.2)$ & $18(4.2)$ & $0.968,0.739-1.268,0.056,0.813^{\ddagger}$ & $1.088,0.553,2.138,0.06,0.807^{\ddagger}$ \\
\hline Male SCZ & $111(62)$ & $63(35.2)$ & $5(2.8)$ & \multirow{2}{*}{$0.941,0.615-1.438,0.08,0.829$} & \multirow{2}{*}{$0.735,0.229-2.359,0.27,0.603^{\S}$} \\
\hline Male controls & $118(63.4)$ & $61(32.8)$ & $7(3.8)$ & & \\
\hline Female SCZ & $154(61.4)$ & $84(33.5)$ & $13(5.2)$ & \multirow{2}{*}{$0.985,0.695-1.398,0.007,0.934$} & \multirow{2}{*}{$1.486,0.64-3.45,0.858,0.354 \S$} \\
\hline Female controls & $174(61.7)$ & $98(34.8)$ & $10(3.5)$ & & \\
\hline EOSCZ & $84(60)$ & $49(35)$ & $7(5)$ & $0.904,0.614-1.331,0.262,0.609$ & $1.396,0.567-3.438,0.532,0.466^{\ddagger}$ \\
\hline LOSCZ & $181(62.4)$ & $98(33.8)$ & $11(3.8)$ & $1.001,0.704-1.354,000,0.995$ & $1.046,0.483-2.266,0.013,0.909^{\ddagger}$ \\
\hline All controls & $292(64.4)$ & $159(34)$ & $17(3.6)$ & & \\
\hline
\end{tabular}

*frequencies of individual alleles (not shown) did not differ significantly between the entire SCZ group or subgroup and healthy controls, ${ }^{\dagger}$ values indicate: odds ratio, $95 \%$ confidence interval, $\chi^{2}$, p value, ${ }^{\ddagger}$ with respect to all controls, ${ }^{\S}$ with respect to the corresponding male or female controls. SCZ: schizophrenia, EOSCZ: early-onset SCZ, LOSCZ: late-onset SCZ

Table 3. Haplotype frequencies at loci rs363371 and rs363324 in Han Chinese with SCZ and healthy controls

\begin{tabular}{ccccccc}
\hline SNP & Haplotype* & SCZ, N (\%) & Control, N (\%) & $\chi^{2}$ & p & OR (95\% CI) \\
\hline \multirow{2}{*}{1,2} & A-G & $470(54.8)$ & $522(56.1)$ & 0.323 & 0.57 & $0.947(0.786-1.142)$ \\
& G-A & $183(21.3)$ & $195(20.9)$ & 0.044 & 0.834 & $1.025(0.816-1.286)$ \\
& G-G & $205(23.9)$ & $214(23.0)$ & 0.216 & 0.642 & $1.053(0.846-1.311)$ \\
\hline
\end{tabular}

*only haplotypes with a frequency of $\mathrm{p}>0.03$ were considered in the analysis. A,G, indicates the allele of rs $363371 ; A, G$ indicates the allele of rs363324. SCZ: schizophrenia, OR: odds ratio, CI: confidence interval

equals 1 with probability (power) 1.0. The Type I error probability associated with this test of this null hypothesis was 0.05 . We used an uncorrected chi-squared statistic to evaluate this null hypothesis.The SPSS 17.0 was used for all statistical analyses. Haplotype analysis was conducted by SHEsis 4.0 online (http://analysis.bio-X.cn/myAnalysis.php).

\section{RESULTS}

From our data, there were no significant differences between SCZ and Controls for gender, age (Table 1). For the SNP rs363371 there were no obvious significant differences of the distribution between the SCZ group and the control group $(\mathrm{OR}=1.607,95 \%$ CI $0.761-1.495, \mathrm{p}=0.709)$ or between the subgroups in dominant model (Table 2). While in the reces- sive model, there was an obvious significant difference between the male SCZ group and the male controls (OR=0.564, 95\% CI 0.357-0.892, $\mathrm{p}=0.014)$. There were not any statistical significant between the SCZ group and the control group, the SCZ female group and controls, the EOSCZ, LOSCZ and the control group (Table 2). For the other SNP rs363324, there were no the obvious differences between the SCZ group and the control group and between the subgroups in both dominant and recessive models (Table 2). For the two SNPs, the allele frequencies were not different in any compared groups (Supplementary Table 2 in the online-only Data Supplement). The linkage disequilibrium D' value between rs363371 and rs363324 was 0.334 , suggesting a strong recombination event. Thus we conduct a Haplotype analysis, in which haplotypes with frequencies $<0.03$ were excluded, identified the three haplotypes 
A-G, G-A, and G-G. None of these haplotypes showed a significant association with SCZ (Table 3).

\section{DISCUSSION}

Our data did not detect an association of schizophrenia with two SNPs in the VMAT2 gene found in PD. Our study was consistent with a previous study that failed to detect an association between VMAT2 and SCZ, nonetheless that study did not including the two SNPs of our study. ${ }^{17}$ Interestingly, we did find the AA genotype of rs363371 as a protective allele for male Han Chinese from developing SCZ ( $\mathrm{OR}=0.564,95 \%$ CI 0.357 to $0.892, \mathrm{p}=0.014$ ). And this locus, located in the promoter of VMAT2, is potentially functional. It is in linkage disequilibrium with locus rs2619096, which doubles the activity of the VMAT2 promoter. ${ }^{18}$

Although many studies have shown a relationship between VMAT2 pathophysiology of neuropsychiatric diseases including schizophrenia, ${ }^{19}$ mood disorders ${ }^{20}$ methamphetamine neurotoxicity. ${ }^{21}$ In patients with SCZ, it was reported that the density of VMAT2 protein increased in platelets, which may indicate a schizophrenia-related hyperactivity of the monoaminergic system or an adaptive response to chronic drug treatment. ${ }^{22}$ In a 14-3-3epsilon hetero KO mice model of schizophrenia, the expression of VMAT2 was increased significantly in the hippocampal, indicating a possible pathophysiology of schizophrenia includes a monoaminergic transmission abnormality. ${ }^{23}$ Recently, a study showed that genetic variation in VMAT2 may be linked to alterations in cognitive functioning underlying psychotic disorder. ${ }^{24}$ VMAT2 SNPs effect on promoter activity or mRNA abundance ${ }^{25,26}$ which affects VMAT2 expression. Based on the above results, we speculated that VMAT2 gene polymorphism may be a risk factor for schizophrenia. Therefore, the study on the association between VMAT2 gene polymorphism and schizophrenia should amplify the sample size, increase the locus and multicenter studies.

Interestingly, our study showed a significant difference of AA genotype of rs363371 between the male patients with SCZ and male controls but not between female patients with SCZ and female controls. It seems contrary to a GWAS research which supports the idea that there is no strong sex-specific genetic risk factors exist for schizophrenia. ${ }^{27}$ But indeed, there were some other evidence supports the potential sex-specific genetic factors. One case-control study showed a gender difference of genetic factors, in which interleukin 1 receptor antagonist protein (IL1Ra) and IGF2BP2 were associated with SCZ in male population but not in female. ${ }^{28,29} \mathrm{C}$ allele and $\mathrm{CC}$ genotype of rs 253 was reported a confer risk for schizophrenia in men. ${ }^{30} \mathrm{~A}$ malespecific association was also be reported with SNP rs62621676 in a UK case-control study. ${ }^{31}$ While, there were more female- specific genetic factors. Two case-control studies from China showed that a high-affinity neurotensin receptor 1 (NTR1) gene polymorphism and estrogen receptor 1 (ESR1) gene polymorphism were increased in the risk of developing SCZ in female Chinese population. ${ }^{32,33}$ A GWAS study found a femalespecific association with rs7341475 in the reelin (RELN) gene. ${ }^{34}$ Most interestingly, a study showed that in the same gene, different SNPs conduct different sex-specific effects, e.g., SNPs rs3891636, rs7728773, rs2189663,rs3864283, and rs2526303 of HINT1 gene were significantly different with controls among the female subjects alone, whereas SNP rs7735116 was significant among males alone. ${ }^{35}$ These studies indicated that sex-specific genetic risk and protective factors may exist in SCZ.

To our knowledge, this is the first case-control study that aimed to detect a potential relationship between SCZ and the two potentially functional SNPs of VMAT2 in Chinese population. Nevertheless, our results have some limitations. First, the relative small sample size may confer a low statistical power (the power for rs 363371 was $80.8 \%$ while for rs 363324 was only 5.1\%). Second, we only selected two SNP in the promoter region, which may have missed other potential risk SNPs. Third, we did not take other risk factors associated with SCZ (e.g., environmental and social factors) into consideration. Moreover, a recent study found that VMAT2 was associated with Tardive dyskinesia (TD), an involuntary movement disorder affecting up to $25 \%$ of patients receiving longterm first-generation antipsychotic treatment. ${ }^{36}$ Thus, the gene-medical treatment should be taken into consideration in the future study.

In conclusions, our study found that rs363371and rs363324 were not associated with SCZ, while it seems that the AA genotype of rs363371 was associated with a protective effect in male Chinese of developing SCZ. In future studies, more SNPs should be included and more risk factors should be involved in a much bigger sample size study recruiting patients from different ethnicities.

\section{Supplementary Materials}

The online-only Data Supplement is available with this article at https://doi.org/10.30773/pi.2020.0023.

\section{Acknowledgments}

We would like to thank Dr. Jihui Zhang, the assistant professor at the department of psychiatry, the Chinese university of Hong Kong for improving the English proficiency throughout the manuscript.

This research was supported by the Science and Technology Program of Guangdong, China (2012B031800357).

\section{Conflicts of Interest}

The authors have no potential conflicts of interest to disclose.

\section{Author Contributions}

Conceptualization: Hongying Han, Xiaowei Xia. Data curation: Huirong 
Zheng, Chongbang Zhao. Formal analysis: Huirong Zheng. Funding acquisition: Jiong Tao, Xianglan Wang. Investigation:Yanming Xu. Methodology: Yanming Xu. Project administration: Jiong Tao, Xianglan Wang. Resources: Jiong Tao. Software: Chongbang Zhao. Supervision: Xianglan Wang. Validation: Jiong Tao, Xianglan Wang. Visualization: Xianglan Wang. Writing_original draf: Hongying Han, Xiaowei Xia. Writing—review \& editing: Jiong Tao, Xianglan Wang.

\section{ORCID iDs}

$\begin{array}{ll}\text { Hongying Han } & \text { https://orcid.org/0000-0003-3254-6158 } \\ \text { Xiaowei Xia } & \text { https://orcid.org/0000-0001-8220-3781 } \\ \text { Huirong Zheng } & \text { https://orcid.org/0000-0002-8548-6921 } \\ \text { Chongbang Zhao } & \text { https://orcid.org/0000-0001-5354-339X } \\ \text { Yanming Xu } & \text { https://orcid.org/0000-0001-7908-235X } \\ \text { Jiong Tao } & \text { https://orcid.org/0000-0002-3572-2084 } \\ \text { Xianglan Wang } & \text { https://orcid.org/0000-0001-8524-3586 }\end{array}$

\section{REFERENCES}

1. Ayhan Y, Sawa A, Ross CA, Pletnikov MV. Animal models of gene-environment interactions in schizophrenia. Behav Brain Res 2009;204:274281.

2. Schizophrenia Working Group of the Psychiatric Genomics Consortium. Biological insights from 108 schizophrenia-associated genetic loci. Nature 2014;511:421-427.

3. Ripke S, O’Dushlaine C, Chambert K, Moran JL, Kahler AK, Akterin S, et al. Genome-wide association analysis identifies 13 new risk loci for schizophrenia. Nat Genet 2013;45:1150-1159.

4. Harrison PJ. Recent genetic findings in schizophrenia and their therapeutic relevance. J Psychopharmacol 2015;29:85-96.

5. Williams NM, Norton N, Williams H, Ekholm B, Hamshere ML, Lindblom Y, et al. A systematic genomewide linkage study in $353 \mathrm{sib}$ pairs with schizophrenia. Am J Hum Genet 2003;73:1355-1367.

6. de Natale ER, Niccolini F, Wilson H, Politis M. Molecular Imaging of the Dopaminergic System in Idiopathic Parkinson's Disease. In: Politis M, Editor. International Review of Neurobiology. Cambridge, Massachusetts: Academic Press, 2018, p.131-172.

7. Brighina L, Riva C, Bertola F, Saracchi E, Fermi S, Goldwurm S, et al. Analysis of vesicular monoamine transporter 2 polymorphisms in Parkinson's disease. Neurobiol Aging 2013;34:1712-1719.

8. Da SAF, Figee M, van Amelsvoort T, Veltman D, de Haan L. The revised dopamine hypothesis of schizophrenia: evidence from pharmacological MRI studies with atypical antipsychotic medication. Psychopharmacol Bull 2008;41:121-132.

9. He X, Zhang L, Yao X, Hu J, Yu L, Jia H, et al. Association studies of MMP-9 in Parkinson's disease and amyotrophic lateral sclerosis. PLos One 2013;8:e73777.

10. Han H, He X, Tang J, Liu W, Liu K, Zhang J, et al. The C(-1562)T polymorphism of matrix metalloproteinase-9 gene is associated with schizophrenia in China. Psychiatry Res 2011;190:163-164.

11. Ira E, Zanoni M, Ruggeri M, Dazzan P, Tosato S. COMT, neuropsychological function and brain structure in schizophrenia: a systematic review and neurobiological interpretation. J Psychiatry Neurosci 2013;38: 366-380.

12. Kiyohara C, Miyake Y, Koyanagi M, Fujimoto T, Shirasawa S, Tanaka $\mathrm{K}$, et al. Genetic polymorphisms involved in dopaminergic neurotransmission and risk for Parkinson's disease in a Japanese population. BMC Neurol 2011;11:89.

13. Carrera N, Sanjuan J, Molto MD, Carracedo A, Costas J. Recent adaptive selection at $\mathrm{MAOB}$ and ancestral susceptibility to schizophrenia. Am J Med Genet B Neuropsychiatr Genet 2009;150B:369-374.

14. First MB, Spitzer RL, Gibbon M, Williams JBW. Structured Clinical Interview for DSM-IV Axis I Disorders, Clinician Version (SCID-CV). Washington, DC: American Psychiatric Press, Inc; 1996.
15. Rapoport JL, Addington AM, Frangou S, Psych MR. The neurodevelopmental model of schizophrenia: update 2005. Mol Psychiatry 2005;10: 434-449.

16. Thomas G, Sinville R, Sutton S, Farquar H, Hammer RP, Soper SA, et al. Capillary and microelectrophoretic separations of ligase detection reaction products produced from low-abundant point mutations in genomic DNA. Electrophoresis 2004;25:1668-1677.

17. Talkowski ME, Kirov G, Bamne M, Georgieva L, Torres G, Mansour $\mathrm{H}$, et al. A network of dopaminergic gene variations implicated as risk factors for schizophrenia. Hum Mol Genet 2008;17:747-758.

18. Lin Z, Walther D, Yu XY, Li S, Drgon T, Uhl GR. SLC18A2 promoter haplotypes and identification of a novel protective factor against alcoholism. Hum Mol Genet 2005;14:1393-1404.

19. Taylor SF, Koeppe RA, Tandon R, Zubieta JK, Frey KA. In vivo measurement of the vesicular monoamine transporter in schizophrenia. Neuropsychopharmacology 2000;23:667-675.

20. Zubieta JK, Taylor SF, Huguelet P, Koeppe RA, Kilbourn MR, Frey KA. Vesicular monoamine transporter concentrations in bipolar disorder type I, schizophrenia, and healthy subjects. Biol Psychiatry 2001;49:110116.

21. Volz TJ, Farnsworth SJ, Rowley SD, Hanson GR, Fleckenstein AE. Agedependent differences in dopamine transporter and vesicular monoamine transporter-2 function and their implications for methamphetamine neurotoxicity. Synapse 2009;63:147-151.

22. Zucker M, Valevski A, Weizman A, Rehavi M. Increased platelet vesicular monoamine transporter density in adult schizophrenia patients. Eur Neuropsychopharmacol 2002;12:343-347.

23. Iritani S, Sekiguchi H, Habuchi C, Hikita T, Taya S, Kaibuchi K, et al. Immunohistochemical study of vesicle monoamine transporter 2 in the hippocampal region of genetic animal model of schizophrenia. Synapse 2010;64:948-953.

24. Simons CJ, van Winkel R. Intermediate phenotype analysis of patients, unaffected siblings, and healthy controls identifies VMAT2 as a candidate gene for psychotic disorder and neurocognition. Schizophr Bull 2013;39:848-856.

25. Li Q, Yan Z, Kuang Y, Zhou X, Jin L, He L, et al. Genetic variations in the 3'-untranslated region of SLC18A2 are associated with serum FSH concentration in polycystic ovary syndrome patients and regulate gene expression in vitro. Hum Reprod 2016;31:2150-2157

26. Bharadwaj RA, Jaffe AE, Chen Q, Deep-Soboslay A, Goldman AL, Mighdoll MI, et al. Genetic risk mechanisms of posttraumatic stress disorder in the human brain. J Neurosci Res 2018;96:21-30.

27. Bergen SE, O'Dushlaine CT, Lee PH, Fanous AH, Ruderfer DM, Ripke S, et al. Genetic modifiers and subtypes in schizophrenia: investigations of age at onset, severity, sex and family history. Schizophr Res 2014;154:48-53

28. Ben NM, Zaabar I, Zaafrane F, Thabet S, Mechri A, Gaha L, et al. A gender-specific association of interleukin 1 receptor antagonist polymorphism with schizophrenia susceptibility. Acta Neuropsychiatr 2013;25:349-355.

29. Zhang X, Hui L, Liu Y, Wang ZQ, You Y, Miao LN, et al. The type 2 diabetes mellitus susceptibility gene IGF2BP2 is associated with schizophrenia in a Han Chinese population. J Clin Psychiatry 2013;74:e287-e292.

30. Xie C, Wang ZC, Liu XF, Wang L, Yang MS. Association between schizophrenia and single nucleotide polymorphisms in lipoprotein lipase gene in a Han Chinese population. Psychiatr Genet 2011;21:307-314.

31. Carroll LS, Williams NM, Moskvina V, Russell E, Norton N, Williams $\mathrm{HJ}$, et al. Evidence for rare and common genetic risk variants for schizophrenia at protein kinase C, alpha. Mol Psychiatry 2010;15:1101-1111.

32. Wang LW, He SP, Zhang JL, Tong DX, He Y, Cheng XL. Association between ESR1 gene polymorphisms and haplotypes with schizophrenia. Zhonghua Yi Xue Yi Chuan Xue Za Zhi 2013;30:21-25.

33. Ma H, Huang Y, Zhang B, Li J, Wang Y, Zhao X, et al. Association between neurotensin receptor 1 (NTR1) gene polymorphisms and schizo- 
phrenia in a Han Chinese population. J Mol Neurosci 2013;50:345-352.

34. Shifman S, Johannesson M, Bronstein M, Chen SX, Collier DA, Craddock NJ, et al. Genome-wide association identifies a common variant in the reelin gene that increases the risk of schizophrenia only in women. PLos Genet 2008;4:e28.

35. Chen Q, Wang X, O'Neill FA, Walsh D, Kendler KS, Chen X. Is the histidine triad nucleotide-binding protein 1 (HINT1) gene a candidate for schizophrenia? Schizophr Res 2008;106:200-207.

36. Zai CC, Tiwari AK, Mazzoco M, de Luca V, Muller DJ, Shaikh SA, et al. Association study of the vesicular monoamine transporter gene SLC18A2 with tardive dyskinesia. J Psychiatr Res 2013;47:1760-1765. 
Supplementary Table 1. Primers used in ligase detection reactions to genotype VMAT2 polymorphisms at rs363371 and rs363324

\begin{tabular}{cclc}
\hline Variant & Base change & \multicolumn{1}{c}{ Primer } & Product (bp) \\
\hline rs363371 & G/A & GATGAACCCAAGGCAGGAAC (forward) & 94 \\
& \multirow{2}{*}{ Gs363324 } & CTCACATGGCACAATGAATG (reverse) & 92 \\
& & CCCTGGAACTAATTCCTGTC (forward) & \\
\hline
\end{tabular}


Supplementary Table 2. VMAT2 allele frequencies in the entire group and subgroups of Han Chinese with SCZ and healthy controls

\begin{tabular}{|c|c|c|c|}
\hline \multirow{2}{*}{ Group or subgroup } & \multicolumn{2}{|c|}{ Allele, N (\%) } & \multirow{2}{*}{ Comparison* } \\
\hline & A & G & \\
\hline \multicolumn{4}{|l|}{ Locus rs363371 } \\
\hline All SCZ & $471(54.8)$ & $389(45.2)$ & $0.945,0.784-1.138,0.550^{\dagger}$ \\
\hline Male SCZ & $195(52.7)$ & $175(47.3)$ & $0.789,0.59-1.054,0.108^{\ddagger}$ \\
\hline Male controls & $219(58.6)$ & $155(41.4)$ & \\
\hline Female SCZ & $276(56.3)$ & $214(43.7)$ & $0.981,0.769-1.253,0.881^{\ddagger}$ \\
\hline Female controls & $318(56.8)$ & $242(43.2)$ & \\
\hline EOSCZ & $146(52.5)$ & $132(47.5)$ & $0.863,0.66-1.129,0.282^{\dagger}$ \\
\hline LOSCZ & $325(55.8)$ & $257(44.2)$ & $0.987,0.801-1.215,0.9^{\dagger}$ \\
\hline All controls & $528(56.2)$ & $412(43.8)$ & \\
\hline \multicolumn{4}{|l|}{ Locus rs363324 } \\
\hline All SCZ & $183(21.3)$ & $677(78.7)$ & $1.041,0.829-1.309,0.723^{\dagger}$ \\
\hline Male SCZ & $73(20.4)$ & $285(76.6)$ & $1.007,0.702-1.446,0.968^{\ddagger}$ \\
\hline Male controls & $75(20.2)$ & $297(79.8)$ & \\
\hline Female SCZ & $110(21.9)$ & $392(78.1)$ & $1.061,0.791-1.422,0.694^{\ddagger}$ \\
\hline Female controls & $118(20.9)$ & $446(79.1)$ & \\
\hline EOSCZ & $63(22.5)$ & $217(77.5)$ & $1.118,0.81-1.542,0.498^{\dagger}$ \\
\hline LOSCZ & $120(20.7)$ & $460(79.3)$ & $1.004,0.778-1.297,0.974^{\dagger}$ \\
\hline All controls & $193(20.6)$ & $743(79.4)$ & \\
\hline
\end{tabular}

*values indicate: OR, 95\% CI, p value, ${ }^{\dagger}$ with respect to all controls, ${ }^{\ddagger}$ with respect to the corresponding male or female controls. SCZ: schizophrenia, EOSCZ: early-onset SCZ, LOSCZ: late-onset SCZ 